\title{
Caracterização das notificações de intoxicações por agrotóxicos na 5 은 Regional de Saúde do Paraná (PR)
}

\author{
Characterization of pesticide intoxications notifications at the 5th Regional Health of \\ Paraná (PR)
}

Caracterización de las notificaciones de intoxicaciones exógenas por pesticidas em la $\mathrm{V}$ Región Regional de Salud de Paraná (PR)

Cristiane de Melo Aggio $^{1,2 *}$, Andriele Leite ${ }^{2}$, Tatiana da Silva Melo Malaquias ${ }^{2}$, Maria do Carmo Fernandez Lourenço Haddad 1 .

\section{RESUMO}

Objetivo: Caracterizar as notificações de intoxicações por agrotóxicos. Métodos: Estudo descritivo, retrospectivo e quantitativo, com análise estatística descritiva dos dados secundários de 20 municípios que

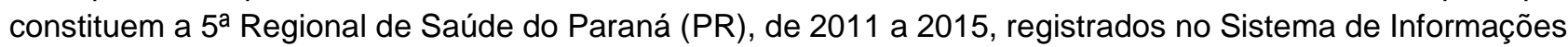
de Agravos de Notificação. Resultados: No período estudado 274 intoxicações por agrotóxicos foram identificadas, sendo observada a média anual de 54,8 notificações e a maioria delas $(75,2 \%)$ ocorreu em cinco municípios. Destacaram-se as vítimas brancas (72,3\%), com idade entre 15 e 56 anos $(86,1 \%)$ e os homens $(73,4 \%)$. Predominaram os acidentes na área rural $(51,4 \%)$, uso acidental de agrotóxicos $(40,1 \%)$, os herbicidas $(60 \%)$, atendimento hospitalar $(60,2 \%)$, a cura sem sequelas $(86,1 \%)$ e a mortalidade masculina $(61,5 \%)$. Conclusão: Os trabalhadores agrícolas do sexo masculino são mais vulneráveis às intoxicações e merecem maior atenção dos gestores, trabalhadores da saúde e pesquisadores para que a proteção da sua saúde seja garantida.

Palavras-chave: Agroquímicos, Envenenamento, Sistemas de informação.

\begin{abstract}
Objective: To characterize notifications of pesticide intoxications. Methods: Descriptive, retrospective and quantitative study, with descriptive statistical analysis of secondary data from 20 municipalities that make up the 5th Regional Health Region of Paraná (PR), from 2011 to 2015, registered in the Notifiable Diseases Information System. Results: During the study period, 274 pesticide poisonings were identified, with an annual average of 54.8 notifications being observed and most of them $(75.2 \%)$ occurred in five municipalities. White victims $(72.3 \%)$, aged between 15 and 56 years $(86.1 \%)$ and men $(73.4 \%)$ stood out. Accidents in the rural area predominated $(51.4 \%)$, accidental use of pesticides $(40.1 \%)$, herbicides $(60 \%)$, hospital care $(60.2 \%)$, cure without sequelae $(86.1 \%)$ and male mortality $(61.5 \%)$. Conclusion: Male agricultural workers are more vulnerable to poisoning and deserve greater attention from managers, health workers and researchers so that the protection of their health is guaranteed.
\end{abstract}

Key words: Agrochemicals, Poisoning, Information systems.

\section{RESUMEN}

Objetivo: Caracterizar las notificaciones de intoxicación por plaguicidas. Métodos: Estudio descriptivo, retrospectivo y cuantitativo, con análisis estadístico descriptivo de datos secundarios de 20 municipios que conforman la V Región Sanitaria Regional de Paraná (PR), de 2011 a 2015, registrados en el Sistema de

1 Universidade Estadual de Londrina (UEL), Londrina - PR. *E-mail: cristianeaggio@gmail.com

2 Universidade Estadual do Centro Oeste (UNICENTRO), Guarapuava - PR.

PUBLICADO EM: 2/2021 
Información de Enfermedades de Notificación. Resultados: Durante el período de estudio se identificaron 274 intoxicaciones por plaguicidas, observándose un promedio anual de 54,8 notificaciones y la mayoría de ellas $(75,2 \%)$ ocurrieron en cinco municipios. Destacaron las víctimas blancas $(72,3 \%)$, de 15 a 56 años $(86,1 \%)$ y hombres $(73,4 \%)$. Predominaron los accidentes en el área rural $(51,4 \%)$, uso accidental de plaguicidas $(40,1 \%)$, herbicidas $(60 \%)$, atención hospitalaria $(60,2 \%)$, curación sin secuelas $(86,1 \%)$. \%) y mortalidad masculina $(61,5 \%)$. Conclusión: Los trabajadores agrícolas varones son más vulnerables a las intoxicaciones y merecen mayor atención por parte de los administradores, trabajadores de la salud e investigadores para garantizar la protección de su salud.

Palabras clave: Agroquímicos, Envenenamiento, Información sistemas.

\section{INTRODUÇÃO}

Os agrotóxicos são substâncias químicas utilizadas na agropecuária brasileira para o controle das pragas danosas à fauna e a flora, as quais possuem propriedades tóxicas prejudiciais ao meio ambiente e à saúde humana. Sua intensa utilização no país ampliou a exposição dos trabalhadores e suas famílias ao envenenamento ou intoxicação, o que compromete a saúde das coletividades e enfraquece suas medidas protetivas, fiscalizatórias e regulatórias (SESA-PR, 2018; DIAS AP, et al., 2018).

O Brasil é um dos maiores consumidores mundiais de agrotóxicos, principalmente na agricultura. Eles também são utilizados no ambiente doméstico para controle de pragas e jardinagem, na medicina veterinária para tratamento de parasitas e na saúde pública para o controle de vetores causadores de doenças. Seu uso ainda se dá no tratamento de madeiras e grãos, produção de flores e na pecuária (SESA-PR, 2018).

Os agrotóxicos podem ser classificados segundo a finalidade como inseticidas, utilizados no extermínio de insetos, herbicidas, para controle de ervas daninha, fungicidas, para controle de fungos, entre outras. Quanto ao grupo químico podem também ser classificados como organofosforados, que são derivados de ácidos e comumente utilizados na agricultura; carbamatos, derivados do ácido carbâmico e utilizados no ambiente doméstico; piretróides, derivados de plantas e utilizados como inseticidas (SESA-PR, 2018).

De acordo com a classificação da Organização Mundial de Saúde a toxicidade dos agrotóxicos está baseada na dose letal de animais de laboratório (DL50) em mg/kg/peso das formulações líquidas e sólidas e sinalizada nas embalagens por meio de diferentes faixas de cores, a saber: classe I (extremamente tóxico e contém faixa vermelha), classe II (altamente tóxico e com faixa amarela), classe III (medianamente tóxico e faixa azul) e classe IV (pouco tóxico e possui faixa verde) (SESA-PR, 2018).

A intoxicações por agrotóxico em seres humanos podem ocorrem de forma intencional (tentativa de suicídio, homicídio ou abortamento), acidental (reutilização de embalagens, fácil acesso das crianças aos produtos), ocupacional (no exercício da atividade do trabalho) e ambiental (água, ar e solo contaminados). Estes agravos podem ser classificados como agudos (decorrente de único ou múltiplos contatos com o agrotóxico em um período de 24 horas, com efeitos que duram de horas a duas semanas), subaguda (com sintomas vagos e subjetivos, que duram horas ou dias) e crônica (surgimento tardio, após meses ou anos de exposição) (SESA-PR, 2018; FIOCRUZ, 2020).

Os trabalhadores agrícolas, pessoas que aplicam agrotóxicos, crianças, mulheres em idade fértil, gestantes e idosos são mais vulneráveis aos seus danos à saúde, como malformações, câncer e lesões em diversos órgãos e sistemas do corpo humano. Como a maioria deles são irreversíveis, este tipo de envenenamento se configura como um problema de saúde pública prioritário (BRASIL, 2018).

O uso frequente dos agrotóxicos pelos trabalhadores agrícolas naturaliza esta prática, banaliza a percepção de risco à saúde e amplia tanto a exposição destas pessoas à intoxicação como a contaminação ambiental (LARA SS, et al., 2019).

A vigilância em saúde das intoxicações por agrotóxicos busca reduzir a morbimortalidade nas populações expostas e, por elas serem um agravo de notificação compulsória, todos os casos suspeitos e confirmados exigem o registro imediato na ficha de notificação padronizada para o Sistema Único de Saúde (SUS) e no 
sistema de informações oficial, pelo profissional de saúde ou responsável pelo serviço que realiza o primeiro atendimento à vítima. Em seguida, tais informações são repassadas para a autoridade de saúde e outras esferas de gestão do SUS (BRASIL, 2018).

Desde 1988 o uso de agrotóxicos por trabalhadores agrícolas é objeto dos estudos científicos e no ano de 2000 ocorreu a ascensão destas publicações, mas, a partir de 2012, a quantidade das produções acadêmicas sobre este tema reduziu, comprometendo a efetivação das práticas de vigilância em saúde do trabalhador e das políticas públicas de saúde (RIBEIRO LP, et al., 2016).

Contudo, os estudos que utilizam dados secundários de fontes oficiais e serviços de saúde sobre as intoxicações por agrotóxico podem auxiliar a avaliação das mudanças nesta morbimortalidade em um dado período e local, além de estimular melhorias das notificações (BRASIL, 2010).

Diante do exposto e para qualificar a atenção à saúde da população exposta aos agrotóxicos, as decisões e ações de prevenção, proteção e promoção são baseadas na análise e interpretação dos dados notificados, logo, o objetivo deste estudo foi caracterizar as notificações de intoxicações por agrotóxicos da $5^{\mathbf{a}}$ Regional de Saúde do Paraná, Brasil, no período de 2011 a 2015.

\section{MÉTODOS}

Realizou-se um estudo descritivo e retrospectivo, com abordagem quantitativa e análise dos dados secundários de notificação de intoxicação exógena por agrotóxicos.

Os municípios da 5ํㅗㄹ Regional de Saúde do Paraná-PR foram a unidade analisada, por serem o cenário das atividades de ensino-aprendizagem dos autores, e os seus dados registrados no Sistema de Informações de Agravos de Notificação (SINAN) constituíram a amostra investigada. Fazem parte da $5^{\mathfrak{a}}$ Regional de Saúde Estado do Paraná os seguintes municípios: Boa Ventura do São Roque, Campina do Simão, Candói, Cantagalo, Foz do Jordão, Goioxim, Guarapuava, Laranjal, Laranjeiras do Sul, Marquinho, Nova Laranjeiras, Palmital, Pinhão, Pitanga, Porto Barreiro, Prudentópolis, Reserva do Iguaçu, Rio Bonito do Iguaçu, Turvo, Virmond.

Como desde 2011 a comunicação das intoxicações se tornou obrigatória (BRASIL, 2011), todos os casos de intoxicação por agrotóxicos registrados entre 2011 e 2015 foram incluídos, inclusive os incompletos. No segundo semestre de 2016 procedeu-se a coleta de dados com consulta ao banco do SINAN. Os dados extraídos da ficha de investigação de intoxicação exógena foram registrados em planilha do Microsoft Excel ${ }^{\circledR}$ e tratados por meio da análise estatística descritiva.

As variáveis de interesse foram: município de ocorrência e ano, características das vítimas (raça autodeclarada, sexo e idade), circunstância da intoxicação (tentativa de suicídio, acidental e ocupacional); classe química do agrotóxico (inseticida, herbicida e outros); tipo de atendimento destes casos e o seu desfecho (óbito e cura com e sem sequela).

Este estudo foi aprovado pelo Comitê de Ética em Pesquisa (COMEP), da Universidade Estadual do Centro Oeste, Guarapuava, Paraná (Parecer no. 1.479.427) e o Termo de Consentimento Livre Esclarecido (TCLE) foi dispensado, por não envolver diretamente seres humanos.

\section{RESULTADOS}

No período de 2011 a 2015 foram documentadas 274 intoxicações por agrotóxicos nos municípios da 5은 Regional de Saúde do Paraná-PR, das quais 22,3\% ocorreram em 2011, 16,8\% em 2012, 18,6\% em 2013, $24,1 \%$ em 2014 e 18,2\% em 2015, sendo observada a média anual de 54,8 notificações.

Dentre os 20 municípios da abrangência da regional de saúde estudada, apenas dois não registraram intoxicações por agrotóxico e a maioria dos casos $(75,2 \%)$ aconteceu em cinco municípios, sendo 54 casos de Prudentópolis, 50 de Laranjeiras do Sul, 49 de Guarapuava, 28 de Pitanga e 25 de Pinhão. Ao longo dos anos, o número de intoxicações por agrotóxicos ascendeu nos municípios de Prudentópolis, Laranjeiras do Sul e Guarapuava, conforme a apresentado a seguir (Figura 1). 
Figura 1 - Número de intoxicações por agrotóxico em cinco municípios $5^{\circ}$ Regional de Saúde, entre os anos 2011 e 2015, Paraná-PR, 2020.

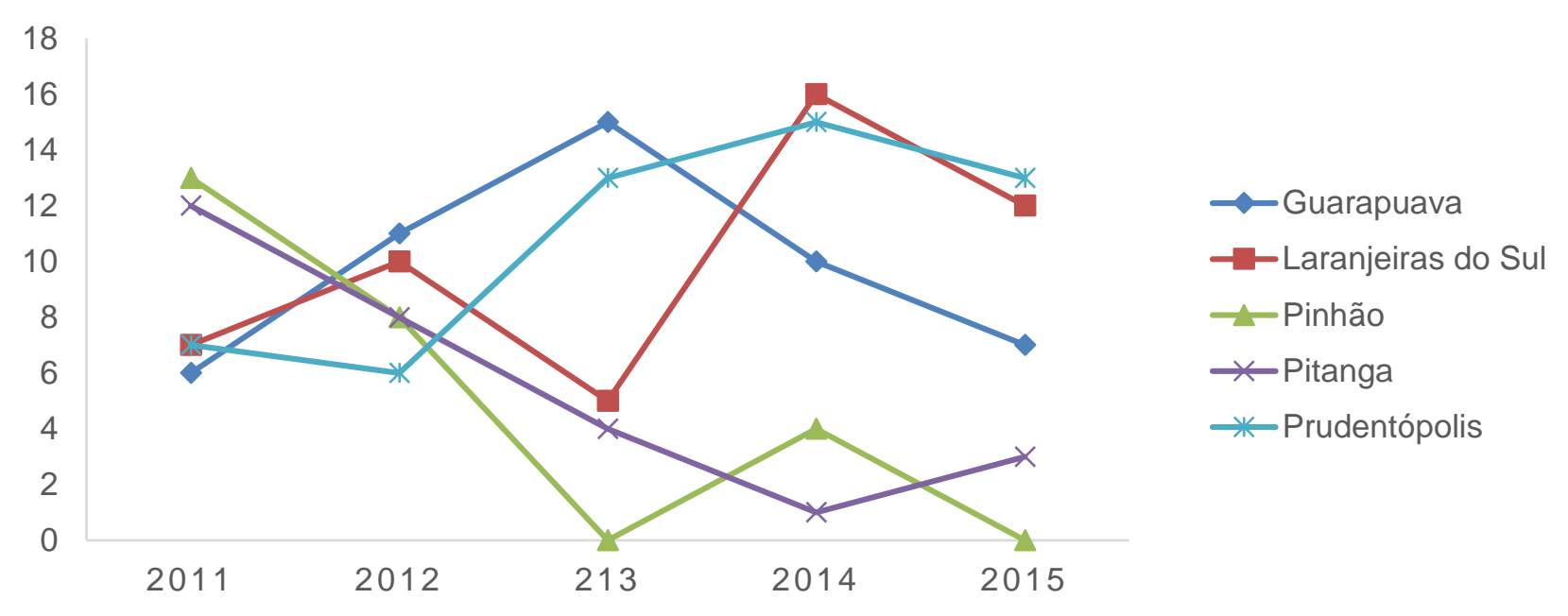

Fonte: Aggio CM, et al., 2020.

A maioria das vítimas de envenenamento por agrotóxico se autodeclarou branca $(72,3 \%)$, era do sexo masculino $(73,4 \%)$ e tinha idade entre 15 e 56 anos (86,1\%). Dentre a população economicamente ativa, detectou-se que $50,4 \%$ das vítimas tinham de 15 a 35 anos de idade e $35,8 \%$ delas tinham idade entre 36 e 56 anos, sendo que $74,2 \%$ desta população era constituída por homens.

Quanto ao local de ocorrência, $51,4 \%$ das intoxicações por agrotóxico se deram na área rural e $24,8 \%$ dos casos não registraram esta informação. O uso acidental de agrotóxicos $(40,1 \%)$, a tentativa de suicídio $(38,7 \%)$ e o uso habitual destas substâncias $(15,7 \%)$ foram as principais circunstâncias em que se deram os envenenamentos notificados.

Verificou-se ainda que os herbicidas $(60 \%)$ e os inseticidas (21\%) foram as principais classes químicas dos agrotóxicos causadores das intoxicações, o hospital foi o serviço de saúde que atendeu $60,2 \%$ das vítimas, a maioria delas evoluiu para a cura sem sequelas $(86,1 \%)$ e, entre os óbitos, os homens predominaram $(61,5 \%)$.

\section{DISCUSSÃO}

A incidência de intoxicação por agrotóxico deste estudo seguramente acompanhou o incremento do uso de pesticidas pelo Brasil entre os anos de 2011 e 2015, o qual conferiu ao país a terceira colocação no ranking das dez economias mundiais que mais utilizaram agrotóxicos, depois dos Estados Unidos e China. A expansão e a modernização da produtividade agrícola, assim como a frágil regulação do uso irresponsável de agrotóxicos, sustentada pelas forças sociais que dela beneficiam, explicam o crescente consumo destas substâncias em nosso país (MORAES RF, 2019).

Além da política desenvolvimentista brasileira incentivadora da produção de bens primários para exportação, a resistência das pragas a alguns herbicidas, o uso autorizado pelos órgãos regulatórios de agrotóxicos anteriormente proibidos, a queda dos preços dos agrotóxicos mais tóxicos e a isenção de alguns tributos favoreceram o aumento da utilização dos agrotóxicos e da exposição dos trabalhadores agrícolas ao envenenamento (PIGNATI WA, et al., 2017).

A agricultura nacional é caracterizada pela produção químico dependente e, desde o período militar, o uso de insumos químicos é estimulado e se intensifica com o passar dos anos, entretanto, muitos dos agrotóxicos utilizados nacionalmente são proibidos em outros países devido a sua toxicidade às pessoas e ao ambiente, a qual provoca mortes, doenças e sofrimento físico e mental (DIAS AP, et al., 2018).

A crescente incidência das intoxicações por agrotóxico observada também ocorreu no Rio Grande do Sul e foi explicada pelo crescimento da agricultura, da comercialização destas substâncias e das ações de 
vigilância em saúde. Todavia o número de intoxicações por agrotóxico de ambos os estudos pode estar subestimado pois para cada caso notificado há outros 50 não identificados. O despreparo dos profissionais de saúde para diagnosticar, notificar e acompanhar estes casos e a insuficiência de estrutura e pessoal nas vigilâncias epidemiológicas municipais podem explicar a subnotificação (FREITAS AB e GARIBOTTI V, 2020; TAVEIRA BLS e ALBUQUERQUE GSC, 2018).

Assim como a desqualificação dos profissionais de saúde para identificar os casos de intoxicação por agrotóxico e estabelecer o nexo causal destes agravos, o assédio das instituições do agronegócio sobre os governos municipais e os fatores políticos de dominação agem sobre a subnotificação dos mesmos, conferindo-Ihes uma "invisibilidade intencional" e privando-os do acesso aos direitos de saúde e seguridade social (PIGNATI WA, et al., 2017).

Além da exposição do trabalhador agrícola aos agrotóxicos, alimentos e moradores das áreas próximas das lavouras podem ser envenenados por meio da pulverização aérea que dispersa estas substâncias, afetando o ambiente circunscrito, e a utilização das unidades de saúde nas épocas de pulverização por pessoas com demandas não programadas (CORRÊA MLM, et al., 2020).

A agricultura familiar está presente em mais de $80 \%$ dos estabelecimentos rurais paranaenses e nacionais e sofre com o ínfimo investimento no setor agropecuário e grande pressão pela produção de alimentos saudáveis. Na região de Laranjeiras do Sul quase metade da população se encontra em pequenas e médias propriedades familiares da área rural que se dedicam ao cultivo de grãos, hortifruticultura e pecuária. A agricultura familiar também predomina na microrregião de Guarapuava, que é composta por 18 municípios que se destacam pela produção de arroz, feijão e mandioca (VICTORIO AM, 2019; SILVA CPO e HAURESKO C, 2014).

Embora alguns agricultores de Prudentópolis estejam cultivando tabaco orgânico, o risco de envenenamento ainda existe e é potencializado pela produção de soja, que é rentável, praticada na entre safra do tabaco e que consome altas quantidades de agrotóxicos. O uso das mãos e de diversos agrotóxicos na fumicultura é decisivo tanto para a produtividade como para o risco de alterações sanguíneas, hepáticas, renais, psiquiátricas, cognitivas, psicomotoras, alergias, esterilidade masculina e cânceres decorrentes das exposições à nicotina e aos agrotóxicos combinados (MURAKAMI Y, et al., 2017; ORGANEK M, 2017).

Como o Brasil é o segundo maior produtor mundial de tabaco, o Paraná é o terceiro maior consumidor de agrotóxicos, quase metade dessas substâncias utilizadas na fumicultura são altamente tóxicos e $75 \%$ dos agrotóxicos utilizados nacionalmente são empregados no cultivo de soja e milho, os profissionais de saúde que atendem os trabalhadores agrícolas devem sempre considerar o diagnóstico diferencial de intoxicações exógenas e notificar todos casos suspeitos, sendo necessário o envolvimento de diversos setores para a prevenção deste agravo (MURAKAMI Y, et al., 2017; FREITAS AB, 2020; BRASIL e, 2019).

O predomínio de intoxicações por agrotóxicos entre os trabalhadores do sexo masculino também foi identificado em uma pesquisa realizada no Rio Grande do Sul e em outras duas desenvolvidas em Goiás possivelmente porque a aplicação destas substâncias é uma atividade realizada predominantemente pelos homens, não podendo ser descartado o risco de envenenamento por mulheres que lavam as roupas e equipamentos de proteção individual contaminados (RISTOW LP, et al., 2020; TEJERINA GRL, 2018; NEVES PDM, et al., 2020).

A tentativa de suicídio, a exposição ocupacional e o acidente individual foram as principais circunstâncias das intoxicações por agrotóxicos deste e de três estudos realizados em Goiás, sendo que o suicídio também foi destacado em outros estados brasileiros, o qual é uma das manifestações psiquiátricas próprias da neurotoxicidade destas substâncias, que é agravada pela exposição crônica e o acesso facilitado a elas (TEJERINA GRL, 2018; NEVES PDM, et al., 2020; OKUYAMA JHH, et al., 2020).

Os herbicidas são frequentemente utilizados nas monoculturas, sendo os agrotóxicos mais comercializados em 2014 e se destacaram entre as substancias responsáveis pelas intoxicações identificadas nesse estudo e, junto ao agente anticolinesterásico carbamato, vulgarmente conhecido como "chumbinho", foram os principais causadores das mortes investigadas em outra pesquisa, na qual os homens, idosos e 
pessoas do setor agropecuário que tentaram suicídio foram os mais suscetíveis à morte por envenenamento (BRASIL, 2018; OKUYAMA JHH, et al., 2020). Como os trabalhadores agrícolas do sexo masculino sobressaíram entre as notificações analisadas nesse estudo e que apresentaram elevada mortalidade urge a implementação de medidas preventivas destas intoxicações.

Dentre as medidas preventivas destaca-se a educação em saúde, sem a qual os trabalhadores agrícolas apresentam conhecimento insuficiente sobre os riscos potenciais da intoxicação por agrotóxicos e o manuseio seguro dos mesmos. Desprovidos das informações de saúde, os trabalhadores agrícolas contam apenas com as orientações proferidas pelo vendedor destas substâncias, que pode não reforçar a importância do uso de EPI para a prevenção do envenenamento por meio do manuseio dos agrotóxicos (VIANNA LCR, et al., 2017; STIVAL LFM e SILVEIRA ALR, 2017).

Os trabalhadores agrícolas de um município de Piauí-PI, com baixa escolaridade, recorriam às informações contidas nos rótulos das embalagens de agrotóxicos e ao conhecimento dos vizinhos para utilizar estas sustâncias, adquiridas em casas agropecuárias e sem receituário agronômico, sendo que a maioria deles as manuseavam sem utilizar EPI. Este caso reitera a importância da educação em saúde para a prevenção dos riscos à saúde dos trabalhadores agrícolas (SANTANA MC, et al., 2016).

A predominância dos atendimentos hospitalares às intoxicações por agrotóxicos averiguada revelou que a atenção primária de saúde (APS) não era o contato preferencial destas pessoas que, provavelmente, buscaram pelo serviço hospitalar quando os sintomas de envenenamento eram intensos, talvez por que elas já estejam familiarizadas com os mesmos, não utilizem os EPI e tenham medo de represália do agronegócio local (TAVEIRA BLS e ALBUQUERQUE GSC, 2018).

Em uma pesquisa realizada com profissionais de saúde de três unidades da Estratégia Saúde da Família do Sul do Brasil foi verificado que os trabalhadores agrícolas recebiam cuidados de saúde voltados para as manifestações agudas dos problemas de saúde, baseado na atenção curativista e no alívio de sinais e sintomas de intoxicação. Este modo de produzir cuidados em saúde elucida a lacuna das ações preventivas da exposição aos agrotóxicos e o despreparo da equipe para realizar as ações de vigilância à saúde dos trabalhadores (MACHADO LM, et al., 2017).

Quando os profissionais de saúde não notificam os casos de intoxicação por agrotóxico e não informam as vítimas sobre os riscos à saúde e formas de prevenir futuros envenenamentos, fica comprometido o controle da morbimortalidade por este agravo, o desenvolvimento de pesquisas e o envolvimento de outros setores e da própria sociedade na legitimação da saúde enquanto direito e do dever estatal de fiscalizar a venda e orientar a utilização desta substância (BRASIL, 2010).

A alta frequência de cura sem sequela verificada entre os casos de intoxicações por agrotóxico se repetiu em Goiás este fato banaliza a manipulação segura destas substâncias pelos trabalhadores agrícolas e compromete a percepção do risco e a vigilância dos seus impactos tardios na saúde que podem ocorrer após meses ou décadas da exposição (NEVES PDM, et al., 2020; RISTOW LP, et al., 2020).

Tais fatos endossam a importância de efetivar as políticas públicas de saúde e capacitar os profissionais da APS para acolher, identificar, notificar e responder às necessidades de saúde e as condições mais prevalentes dos trabalhadores agrícolas, de modo a superar a assistência descontinua que lhes é oferecida e que traduz a dificuldade do sistema público de saúde nacional em efetivar o modelo de atenção integral em saúde (DIAS AP, et al., 2018; MIRANDA SVC, et al., 2020; VIANNA LCR, et al., 2017).

A intoxicação por agrotóxico evidenciada é um problema público local e nacional cujo conhecimento é primordial ao planejamento, implementação, monitoramento e avaliação da comercialização segura destas substâncias, da promoção da saúde do trabalhador e do homem, além da longitudinalidade do cuidado pela Atenção Primária à Saúde (APS).

A exposição aos agrotóxicos e aos fatores climáticos e ambientais, como a temperatura e a luminosidade solar, são determinantes ambientais da saúde e a interferência dos mesmos nas condições de vida, trabalho e saúde dos trabalhadores agrícolas deve ser identificada, prevenida e assistida pelos agentes comunitários de saúde e demais profissionais de saúde sempre que eles buscarem por atendimento, além de avaliar os 
riscos organizacionais e mecânicos dos mesmos, sobretudo o uso ou não dos EPI (KOFLER I e PANDOLFI MAC, 2019; VIANNA LCR, et al., 2017).

Investir na capacitação dos profissionais da Rede de Atenção à Saúde é uma ação prioritária, já que eles assumem as atribuições da vigilância em saúde do trabalhador e o Centro de Referência Regional em Saúde do Trabalhador (CEREST) nos diversos municípios em que eles não estão implantados. Essa incompletude das ações de vigilância à saúde também se dá pelo desinteresse de alguns municípios em fiscalizar a comercialização dos agrotóxicos e pela dificuldade de outros em disponibilizar as amostras ambientais e humanas suficientemente nas redes de laboratórios públicos para o monitoramento regular das amostras de água, resíduos de agrotóxicos em amostras de ar, alimentos e material biológico humano (CORREA MLM, et al., 2020).

Os resultados deste estudo favoreceram a visibilidade dos riscos e agravos à saúde dos trabalhadores do território de uma Regional de Saúde. Uma limitação a ser considerada é a falta de informações preenchidas nas fichas de notificação de intoxicação exógena consultadas, como a ocupação, via de exposição e grupo do agente tóxico.

Novas pesquisas podem explorar a intoxicação do meio ambiente e alimentos, as perspectivas dos trabalhadores agrícolas e da saúde sobre o manuseio seguro dos agrotóxicos, ampliar a série histórica de comparação da incidência destas notificações, averiguar as relações causa-efeito deste envenenamento, detalhar as características destes territórios, suas atividades produtivas, movimentos sociais e instituições atuantes.

\section{CONCLUSÃO}

A vulnerabilidade dos trabalhadores agrícolas foi a principal característica da população intoxicada por agrotóxicos notificada e estudada, em particular a exposição dos homens jovens às intoxicações por herbicidas e inseticidas, a qual sofre com a insipiente atuação da APS no seu cuidado, o despreparo dos profissionais de saúde para a sua vigilância e a fragilidade das políticas públicas em preveni-la. Tais aspectos devem ser considerados por gestores, trabalhadores da saúde e pesquisadores para efetivar a proteção da saúde desta população.

\section{REFERÊNCIAS}

1. BARBOSA LR. Uso de agrotóxicos e seus impactos na saúde humana e ao meio ambiente: um estudo com agricultores da micro bacia hidrográfica do Ribeirão Arara no Município de Paranavaí, PR. Monografia (Especialização em Gestão Ambiental em Municípios) - Universidade Tecnológica Federal do Paraná, Medianeira, 2014; 41 p.

2. BRASIL. Portaria no 104, de 25 de janeiro de 2011. Define as terminologias adotadas em legislação nacional, conforme o disposto no Regulamento Sanitário Internacional 2005 (RSI 2005), a relação de doenças, agravos e eventos em saúde pública de notificação compulsória em todo o território nacional e estabelece fluxo, critérios, responsabilidades e atribuições aos profissionais e serviços de saúde. Diário Oficial da União, Brasília, DF, n. 18, 26 jan. 2011b. Seção 1, p. 37. Disponível em: https://bvsms.saude.gov.br/bvs/saudelegis/gm/2011/prt0104_25_01_2011.html. Acesso em: 05 de nov de 2020.

3. BRASIL. MINISTÉRIO DA SAÚDE (BRA). Agrotóxicos na ótica do Sistema Único de Saúde. 2v. Brasília: Ministério da Saúde, 2018. 193 p.

4. BRASIL. MINISTÉRIO DA SAÚDE (BRA). Diretrizes para vigilância em saúde de populações expostas a agrotóxicos. [S. I.]; Ministério da Saúde, 2010. 13 p.

5. BRASIL. MINISTÉRIO DA SAÚDE (BRA). Agrotóxicos na ótica do Sistema Único de Saúde. Brasília: Ministério da Saúde, 2019. 250 p.

6. CORREA MLM, et al. AGROTÓXICOS, SAÚDE E AMBIENTE: ação estratégica e políticas públicas em territórios do agronegócio. Rev. Políticas Pública, 2020;24(1):1-17.

7. DIAS AP, et al. Agrotóxicos e saúde. Rio de Janeiro: Fiocruz, 2018. 120 p. (Série Fiocruz - Documentos Institucionais; 2). Coleção Saúde, Ambiente e Sustentabilidade.

8. FREITAS AB, GARIBOTTI V. Caracterização das notificações de intoxicações exógenas por agrotóxicos no Rio Grande do Sul, 2011-2018. Epidemol Serv Saúde [no prelo], 2020.

9. FUNDAÇÃO OSVALDO CRUZ (FIOCRUZ). Sistema Nacional de Informações Tóxico-Farmacológicas - SINITOX. Disponível em: https://sinitox.icict.fiocruz.br/. Acesso em: 13 de nov de 2020.

10. KOFLER I, PANDOLFI MAC. Condições de trabalho do trabalhador rural avaliando o impacto da exposição ao agrotóxico. Interface Tecnológica, 2019;16(1):460-9. 
11. LARA SS, et al. A agricultura do agronegócio e sua relação com a intoxicação aguda por agrotóxicos no Brasil. Hygeia - Revista Brasileira de Geografia Médica e da Saúde, 2019;15(32):1-19.

12. MACHADO LM, et al. Atuação dos profissionais de saúde da família frente ao trabalhador rural exposto a agrotóxicos. Ciênc. Cuid Saúde, 2017;16(3):1-8.

13. MIRANDA SVC, et al. Necessidades e reivindicações de homens trabalhadores rurais frente à atenção primária à saúde. Trabalho, Educação e Saúde, 2020;18(1).

14. MORAES RF. 2506 Agrotóxicos no Brasil: padrões de uso, política da regulação e prevenção da captura regulatória. Brasília: Instituto de Pesquisa Econômica Aplicada, 2019. 76 p.

15. MURAKAMI Y, et al. Intoxicação crônica por agrotóxicos em fumicultores. Saúde Debate, 2017;41(113):563-76.

16. NEVES PDM, et al. Intoxicação por agrotóxicos agrícolas no estado de Goiás, Brasil, de 2005-2015: análise dos registros nos sistemas oficiais de informação. Ciênc Saúde Coletiva, 2020;2(7):2743-54.

17. OKUYAMA JHH, et al. Intoxicações e fatores associados ao óbito por agrotóxicos: estudo caso controle, Brasil, 2017. Rev Bras Epidemiologia, 2020; 23:e200024.

18. ORGANEK M. A produção de tabaco pela agricultura camponesa na comunidade de Linha Piquiri, Prudentópolis (PR). Trabalho de conclusão de curso (Licenciatura Interdisciplinar em Educação do Campo) - Universidade Federal da Fronteira Sul, Laranjeiras do Sul, Paraná, 2017. 58 p.

19. PIGNATI WA, et al. Distribuição espacial do uso de agrotóxicos no Brasil: uma ferramenta para a Vigilância em Saúde. Ciênc. Saúde Coletiva, 2017;22(10):3281-3293.

20. RIBEIRO LP, et al. Trabalho rural, uso de agrotóxicos e adoecimento: um estudo bibliométrico. Rev Med Minas Gerais, 2016; 26 (Supl 8): S318-23.

21. RISTOW LP, et al. Fatores relacionados à saúde ocupacional de agricultores expostos a agrotóxicos. Saúde e Sociedade, 2020; 29(2): e180984.

22. SANTANA MC, et al. Exposição ocupacional de trabalhadores rurais a agrotóxicos. Cad. Saúde Colet., 2016; 24(3):301-7.

23. SECRETARIA DA SAÚDE DO ESTADO DO PARANÁ (SESA-PR). Material técnico de intoxicações agudas por agrotóxicos - atendimento inicial do paciente intoxicado. Paraná: Governo do Estado do Paraná, 2018.120 p.

24. SILVA CPO, HAURESKO C. A participação da agricultura familiar na produção agrícola da microrregião de Guarapuava, no Centro-Sul do Paraná. In: Semana de Iniciação Científica, 19, 2014, Guarapuava. Anais ... Guarapuava: Universidade Estadual do Centro Oeste, 2014.

25. STIVALL FM, SILVEIRA ALRS. Influência da intoxicação por agrotóxicos na saúde do trabalhador rural brasileiro. Revista Eletrônica de Educação da Faculdade Araguaia, 2017;11:245-60.

26. TAVEIRA BLS, ALBUQUERQUE GSC. Análise das notificações de intoxicações agudas, por agrotóxicos, em 38 municípios do estado do Paraná. Saúde em Debate, 2018;42(4):211-22.

27. TEJERINA GRL. Intoxicações e óbitos por agrotóxicos no Estado de Goiás, Brasil, e inovações legislativas. Cad IberoAmer Dir Sanit., 2018; 7(1): 229-49.

28. VIANNA LCR, et al. Vigilância em Saúde do Trabalhador: um estudo à luz da Portaria no 3.120/98. Saúde em Debate, 2017; 41(114): 786-800.

29. VICTORIO AM. Diagnóstico da aquicultura na região de laranjeiras do sul-PR: estado atual e perspectivas. Dissertação (Mestrado em Agroecologia e Desenvolvimento Rural Sustentável) - Universidade Federal da Fronteira Sul, Laranjeiras do Sul, 2019. 61 p. 